\author{
EDUARDO PINTO DE FIGUEIREDO \\ JOÃO BATISTA DO NASCIMENTO \\ MARCO AURÉLIO FERREIRA ADÃO \\ OZÉIAS LOPES
}

\title{
SMART BOX - UMA FORMA DE DIGITALIZAR DADOS DE PROCESSO E AUXILIAR NO SET UP DE MOINHOS ANTIGOS DE TINTA A PÓ
}




\title{
EDUARDO FIGUEIREDO \\ JOÃO BATISTA DO NASCIMENTO \\ MARCO AURÉLIO FERREIRA ADÃO \\ OZÉIAS LOPES
}

\section{SMART BOX - UMA FORMA DE DIGITALIZAR DADOS DE PROCESSO E AUXILIAR NO SET UP DE MOINHOS ANTIGOS DE TINTA A PÓ}

\author{
Monografia apresentada como resultado \\ parcial à obtenção do grau de Especialista em \\ Engenharia Industrial 4.0. Curso de Pós- \\ graduação Lato Sensu, Setor de Tecnologia, \\ Departamento de Engenharia Mecânica, \\ Universidade Federal do Paraná.
}

Orientador: Prof. Dr. Alessandro Marques

\section{CURITIBA}




\section{RESUMO}

O objetivo deste trabalho é o de validar, através de observação, registro e resultados práticos (estudo de caso), que um sistema eletrônico de coleta e digitalização de dados do processo e do ambiente - em máquinas antigas que não possuem recursos de comunicação - pode garantir ganho de produtividade através de melhor setup destas máquinas. A ideação é que o dispositivo, à medida que os dados são extraídos, possa ser realimentado para estabelecer limites operacionais ótimos e transmiti-los através de uma interface simples e amigável para que o operador e/ou gestor responsável possa monitorar e tomar decisão sobre a melhor forma de produzir naquele exato momento, respeitando-se os limites de máquina. Este trabalho é focado em máquinas tipo Moinhos de Sistema de Impacto para tinta a pó. A solução idealizada foi chamada de Smart Box, dispositivo composto por um conjunto de elementos tecnológicos de hardware e software altamente flexível, adaptável a vários tipos de processos, permite coleta, processamento dos dados e interfaces intuitivas via dispositivos móveis através de uma conexão de rede do tipo LAN ou GSM.

Palavras-chave: Moagem por impacto. Powder Coating. Micronização, Digitalização de Processos, Monitoramento em tempo real. 


\section{LISTA DE ILUSTRAÇÕES}

FIGURA 1 - DIAGRAMA DE BLOCOS FUNCIONAL SIMPLIFICADO DO MOINHO.............. 1 FIGURA 2 - CURVA GRANULOMÉTRICA - EQUIPAMENTO MALVERN MASTERSIZER

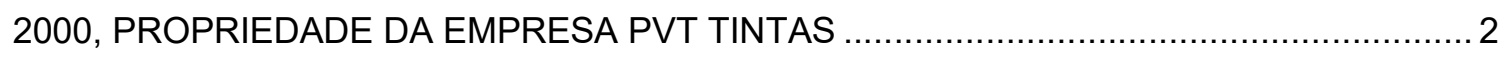

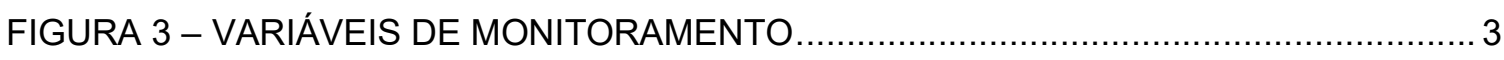

FIGURA 4 - FASE DO DESENVOLVIMENTO DE UM PROJETO DE INTELIGÊNCIA

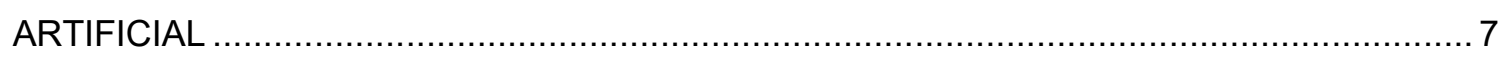

FIGURA 5 - EXEMPLOS DE DASHBOARD PARA SMART BOX $\ldots \ldots \ldots \ldots \ldots \ldots \ldots \ldots \ldots \ldots \ldots . . . . \ldots$

FIGURA 6 - GRÁFICO DE ANÁLISE TOTAL DA PRODUTIVIDADE VS. SETUP DE

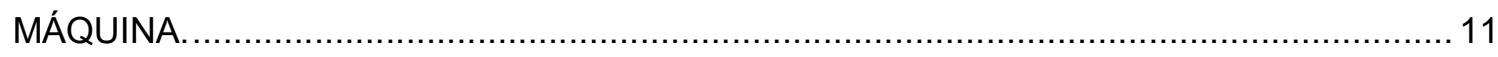

\section{LISTA DE TABELAS}

TABELA 1 - MÊS DE FEVEREIRO DE 2021, COM O SEGUNDO LOTE SENDO OBJETO DE ESTUDO.

TABELA 2 - MÊS DE ABRIL DE 2020: DOIS LOTES, DIFERENTES SETUPS, DIFERENTES RESULTADOS

TABELA 3 - GANHO DE 59,03\% DE PRODUTIVIDADE E 32,16\% DE GANHO NA PERDA DE MATERIAL 


\section{CONTEÚDO}

1. INTRODUÇÃO

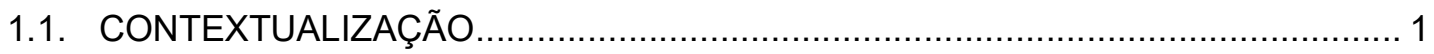

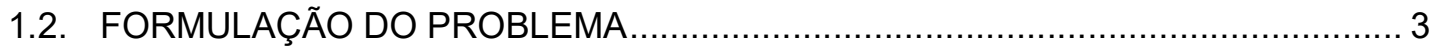

1.3. JUSTIFICATIVA .

1.4. HIPÓTESE

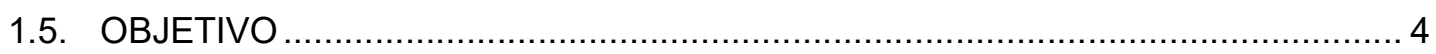

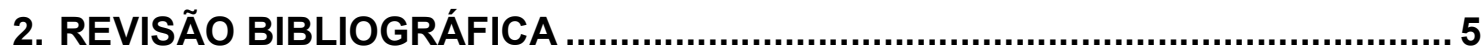

2.1. HARDWAR PRINCIPAL

2.2. SENSORES .

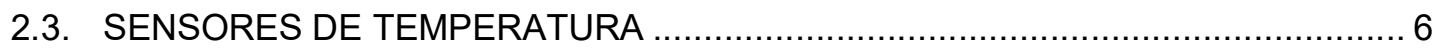

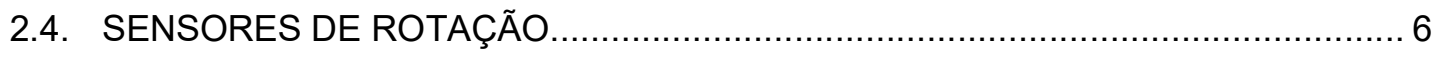

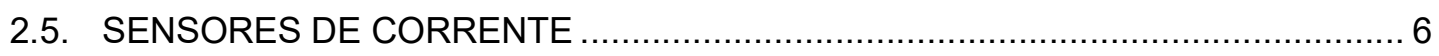

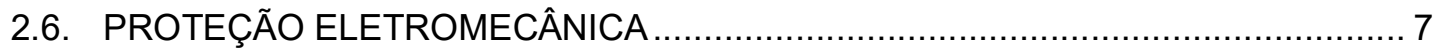

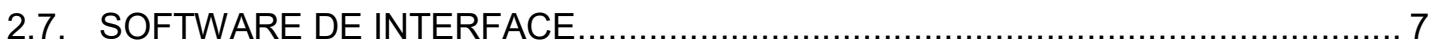

3. METODOLOGIA E PLANEJAMENTO EXPERIMENTAL.................................. 8

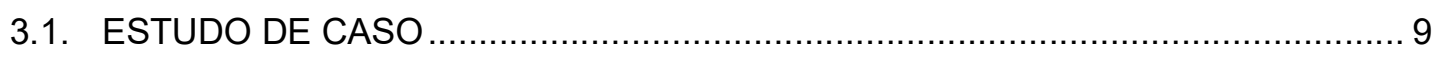

3.2. PROJETO: LEVANTAMENTE E OBSERVAÇÃO ………............................... 9

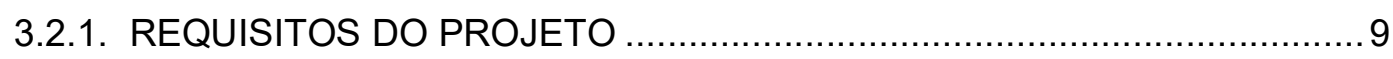

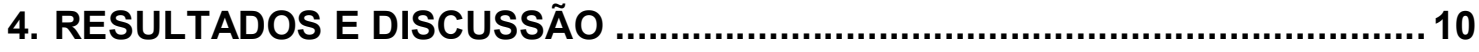

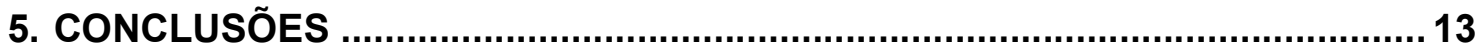

6. REFERÊNCIAS BIBLIOGRÁFICAS.......................................................... 14

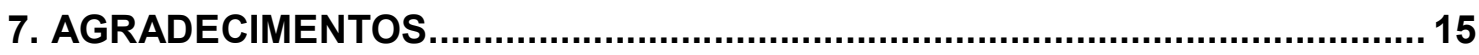





\section{INTRODUÇÃO}

\subsection{CONTEXTUALIZAÇÃO}

Moagem é um processo pelo o qual o material a ser moído passa por um sistema de impacto (Misev, 1977), corte ou atrito. Este sistema busca através de uma destas três grandezas atingir o tamanho de partícula ideal para determinada aplicação. Para atingir o tamanho de partícula ideal independente do processo utilizado, aplica-se um estudo da distribuição e dimensões da partícula denominada granulometria. Durante o processo de moagem existem várias grandezas que alteram a curva granulométrica e produtividade do produto. Hoje em grande parte dos moinhos instalados aplica-se o conhecimento do fornecedor do equipamento ou do próprio operador sobre as variáveis de processo, não havendo assim estabilidade e garantia de melhor rendimento/qualidade do produto. A Figura 1 representa o diagrama de blocos simplificado do sistema.

\section{FIGURA 1 - DIAGRAMA DE BLOCOS FUNCIONAL SIMPLIFICADO DO MOINHO}

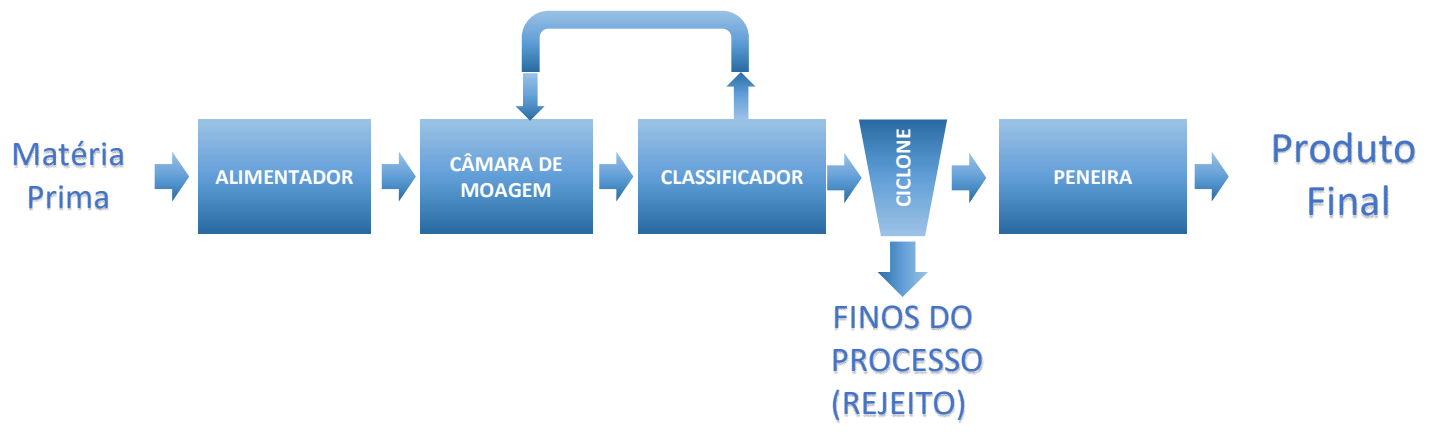

FONTE: AUTORES (2021)

A matéria prima é inserida através do alimentador e segue até a câmara de moagem que possui um disco de moagem, este tem a função de acelerar o material fazendo com que ele bata contra a parede corrugada da câmara de moagem (banda de moagem) de modo continuo, ao se chocar contra a parede ele é quebrado em diversos pedaços menores, essas partículas menores por sua vez são direcionados e sugados pelo ar (regulagem constante neste estudo) contra o classificador, o qual a velocidade é controlada e gera uma barreira física limitando a passagem de material 
moído pelo tamanho de partícula. O material mais "grosso" não consegue passar retornando ao disco de moagem e iniciando novamente o processo. Este processo de quebra gera um aquecimento do material devido ao atrito, e acaba gerando uma carga maior para o motor principal e para o classificador devido a soma do material que está sendo alimentado e o material que está sendo reprocessado, importante destacar que o excesso de reprocessamento poderá gerar granulometrias muito finas que serão sugadas pelo filtro e são perdas de processo. Após o material sair da câmara de moagem ele passa pelo ciclone o qual serve para retirar a parte fina do pó, este material é utilizado para gerar uma curva granulométrica mais fechada, conforme ilustrado na Figura 2, melhorando assim a qualidade do produto.

\section{FIGURA 2 - CURVA GRANULOMÉTRICA - EQUIPAMENTO MALVERN MASTERSIZER 2000, PROPRIEDADE DA EMPRESA PVT TINTAS.}

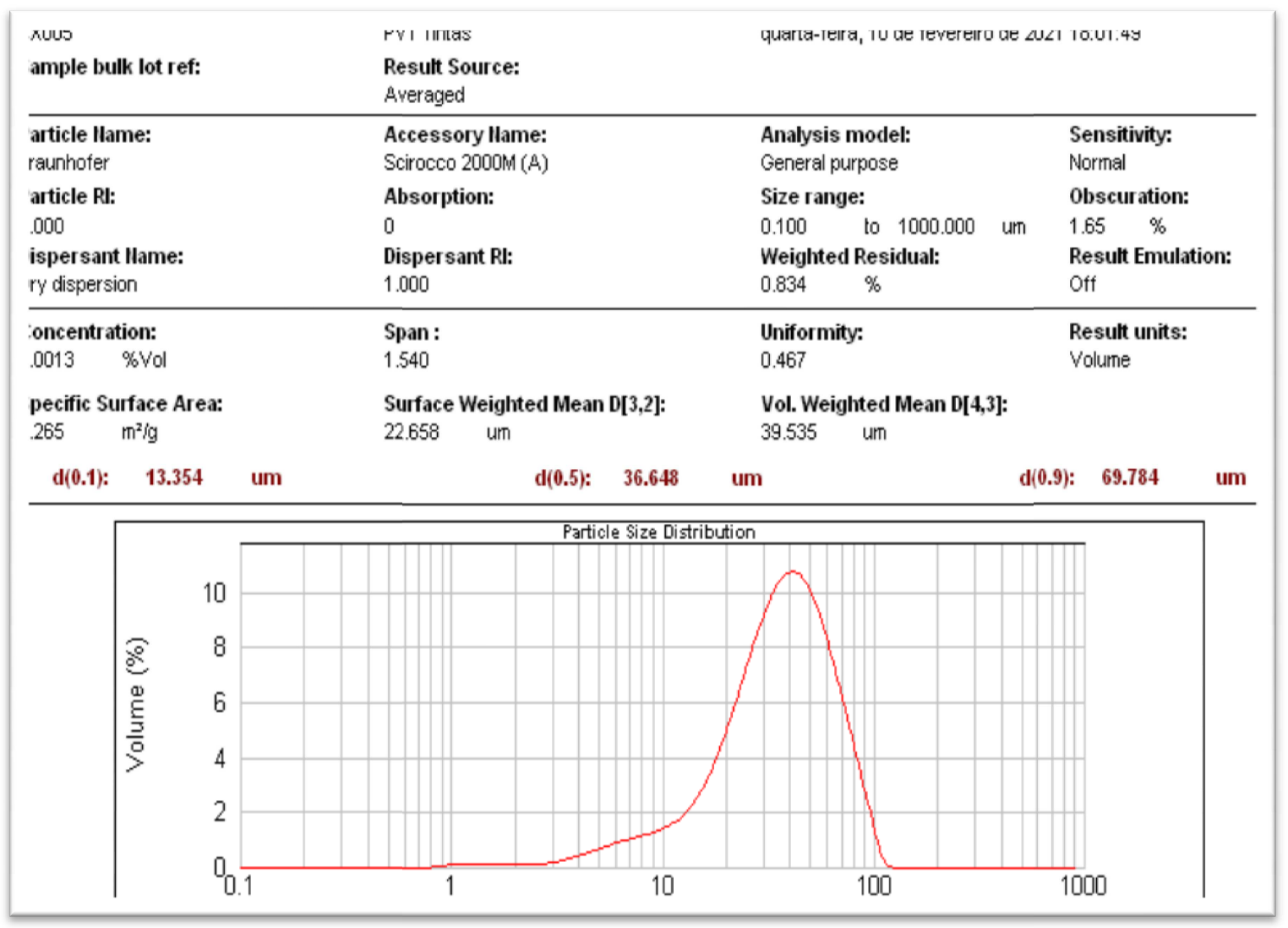

FONTE: AUTORES (2021) 


\subsection{FORMULAÇÃO DO PROBLEMA}

A temperatura de saída do material dever respeitar limite de transição vítrea de cada material (Paiva, et al. 2006), sob risco elevado de compactar o pó formando grumos que são inaceitáveis no produto. Portando uma boa relação entre o motor do classificador, velocidade do ar e motor do alimentador é essencial para um bom aproveitamento do moinho. As variáveis quando controladas podem gerar menos perdas e maior produtividade do equipamento, ou seja, pode-se melhorar desde que se possa monitorar, analisar e atuar sobre o setup da máquina. Das observações operacionais e inter-relações do processo foram determinadas as variáveis a serem monitoradas, com a premissa de respeitar o modo de operação atual do sistema. A Figura 3 apresenta as principais variáveis de controle a serem monitoradas.

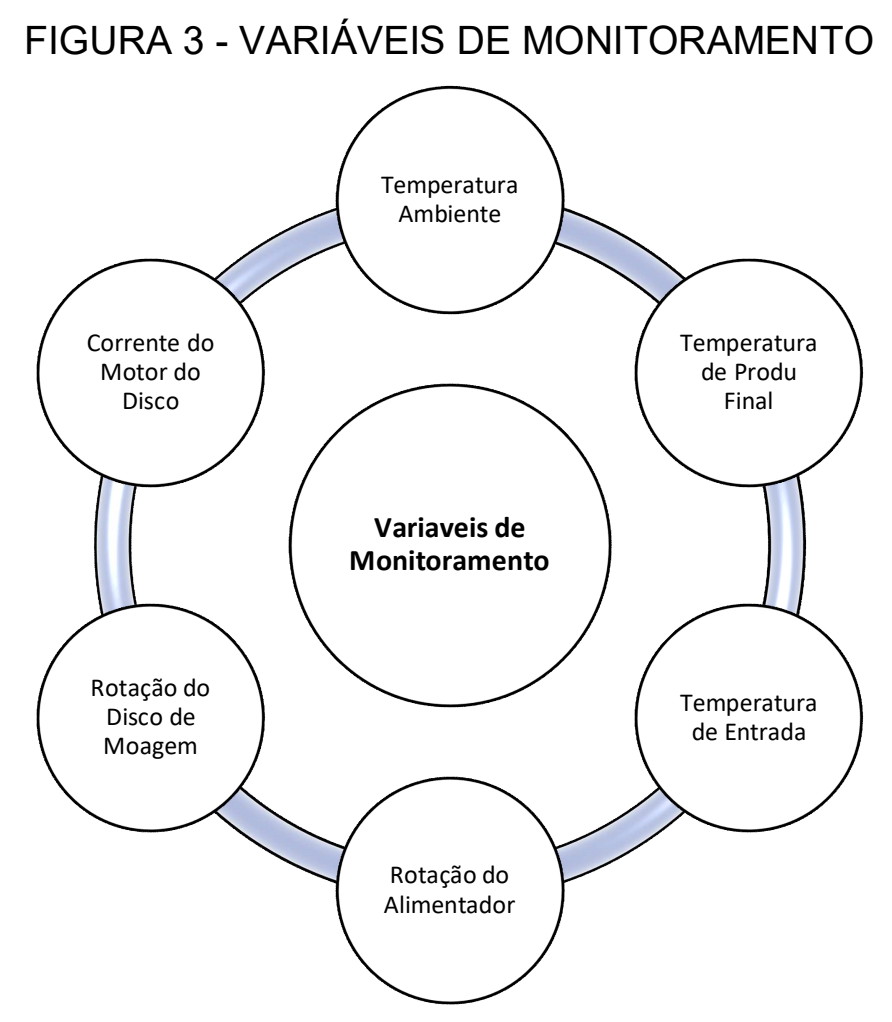

FONTE: AUTORES (2021) 


\subsection{JUSTIFICATIVA}

Após diversas análises e acompanhamento dos moinhos, foi verificado que muitas das variações de qualidade e produtividade estão ligadas à interferência humana no processo. O conhecimento específico de cada operador, o tempo de reação, o "bem-estar" são algumas das influências possíveis no processo, uma vez que fica "nas mãos" do operador determinar o momento certo para fazer um ajuste no processo e melhorar a produtividade da máquina ou simplesmente garantir a qualidade mínima do produto.

\subsection{HIPÓTESE}

Como solução para este cenário, e baseados nos conceitos mineração de dados (Avelar, Cátia Fabíola, 2017) de processos para análises e monitoramento remotos, foi proposto um equipamento que possa realizar a coleta, tratamento e transferências de dados do processo em tempo real, gerando históricos e alarmes, ao qual denominada Smart Box.

\subsection{OBJETIVO}

O Smart Box utiliza soluções simples de hardware para garantir em um primeiro momento a coleta de dados do processo, e após definido os limites de controle pelo especialista passa a ser a interface simplificada e gráfica para auxiliar na tomada de decisões no controle da performance da máquina, baseado em dados estatísticos e técnicos, uma vez que o Smart Box compara todas as grandezas que interferem no processo e definido os limites indica ao operador quando alguma variável está fora do intervalo parametrizado, visa estabelecer a convergência do padrão estabelecido e consequente melhoria da performance da máquina, produtividade e a qualidade necessária do produto final. 


\section{REVISÃO BIBLIOGRÁFICA}

\subsection{HARDWARE PRINCIPAL}

Buscando a melhor solução e entre diversas opções dentro da indústria 4.0 optou-se pelo microprocessador Arduino Uno R3 (Eridani, 2018), uma placa baseada no microcontrolador Tmega328. Ele tem 14 pinos de entrada/saída digital (dos quais 6 podem ser usados como saídas PWM), 6 entradas analógicas, um cristal oscilador de $16 \mathrm{MHz}$, uma conexão USB, uma entrada de alimentação uma conexão ICSP (In Circuit Serial Programming) e um botão de reset. A escolha deste modelo viabiliza todo o conceito do sistema, pois garante uma flexibilização e o baixo custo do produto, além de ser uma ferramenta com uma vasta opção de acessórios.

Para garantir a comunicação do sistema, apesar da placa do Arduino possuir uma conexão de rede Ethernet, foi utilizado um módulo de comunicação GSM SIM 900. Este módulo consegue conectar o Arduino a rede GSM (Sistema Global para Comunicações Móveis), isto faz com que o Smart Box se torne mais funcional e ofereça suporte a cliente que não optem por conectar o mesmo em sua rede, uma vez que o funcionamento é similar ao de um celular, capaz de estabelecer acesso remoto independente da rede do cliente. O módulo vem equipado com um slot para cartão SIM compatível com todas as operadoras de telefonia celular e com duas entradas P2, uma para microfone e outra para reprodução de áudio.

\subsection{SENSORES}

Para garantirmos o correto funcionamento do Smart Box e a precisão dos dados coletados, alguns sensores são necessários para fazer a interface com a máquina. Como o foco da aplicação do dispositivo é em máquinas antigas e usualmente instaladas em ambientes industriais, optou-se pela especificação de sensores utilizados na indústria (Thomazini, 2020). Contudo, as especificações mecânicas são em geral simples de usar e de se conectar as partes das máquinas em questão. 


\subsection{SENSOR DE TEMPERATURA}

A variação da temperatura é um dado importante e necessário para garantir o controle do processo. Foi especificado um termômetro digital modelo DS18B20 que possui sua própria inteligência. Ele é capaz de ler a temperatura, interpretá-la e enviar a informação do valor de temperatura em graus Celsius para o microcontrolador usando um barramento de apenar um fio (protocolo de comunicação One wire ou 1wire). O sensor de temperatura DS18B20 pode medir temperaturas entre $-55^{\circ} \mathrm{C}$ e $125^{\circ} \mathrm{C}$, com uma precisão de cerca de $0,5^{\circ} \mathrm{C}$ na faixa de $-10{ }^{\circ} \mathrm{C}$ e $+85^{\circ} \mathrm{C}$, que atende ao intervalo de trabalho de $-5^{\circ} \mathrm{C}$ a $+60^{\circ} \mathrm{C}$ da planta deste estudo.

Para o caso específico será utilizado sensor tipo sonda e ligação em barramento coletando os dados da temperatura ambiente, entrada e saída do processo.

\subsection{SENSOR DE ROTAÇÃO}

Atualmente o processo é controlado usando como base os dados de velocidade do conversor de frequência do acionamento principal, porém, seria necessária uma interface eletrônica para comunicar com este drive e, em alguns casos, a substituição do mesmo por um modelo atualizado que permitisse algum modo de comunicação. Considerando a necessidade de monitorar esta grandeza, foi escolhido um sensor do tipo Encoder modelo 9SS28 de rotação conectado no eixo principal das máquinas e conectado na entrada digital do Arduino, garantindo assim o monitoramento da rotação atual da máquina.

\subsection{SENSOR DE CORRENTE}

Através da corrente elétrica do acionamento principal, é possível detectar o esforço que a máquina exerce sobre as partículas de tinta, e quanto mais alto este valor, maior o esforço e maior a temperatura, gerando assim uma perda na qualidade 
do produto. Para garantir este monitoramento foi escolhido um sensor de corrente modelo SCT-013-000 com faixa de medição até 100A.

\subsection{PROTEÇÃO ELETROMECÂNICA DO HARDWARE}

Levando em consideração que o Smart Box será instalado em ambientes industriais, foi previsto a montagem do processador e acessórios em caixa com isolação mecânica de alumínio e o uso de fonte de alimentação com isolação galvânica, bem como relés de interface, afim de se evitar danificação por choques mecânicos ou partículas, assim como interferências elétricas ou eletromagnéticas.

\subsection{SOFTWARE DE INTERFACE}

A concepção de algo artificial que simula a inteligência humana ou algo próximo a isso, tem sido motivo de discussão há muito tempo na história. $O$ Aprendizado de Máquina envolve um método de avaliação de dados que automatiza o desenvolvimento de padrões analíticos. Tem como base a concepção de que sistemas tecnológicos podem aprender usando dados, de modo a descobrir padrões, tomar decisões e se aperfeiçoar com pouca interferência humana. Dessa forma, pode melhorar a realização de uma atividade ao longo do tempo.

Mapear o processo, identificar as atividades críticas e problemas que possam melhorar o uso da inteligência artificial é uma fase importante para garantir a correto funcionamento do sistema. A Figura 4 apresenta as fases normalmente aplicadas no desenvolvimento de projetos de inteligência artificial (Rosa, 2011).

FIGURA 4 - FASE DO DESENVOLVIMENTO DE UM PROJETO DE INTELIGÊNCIA ARTIFICIAL 


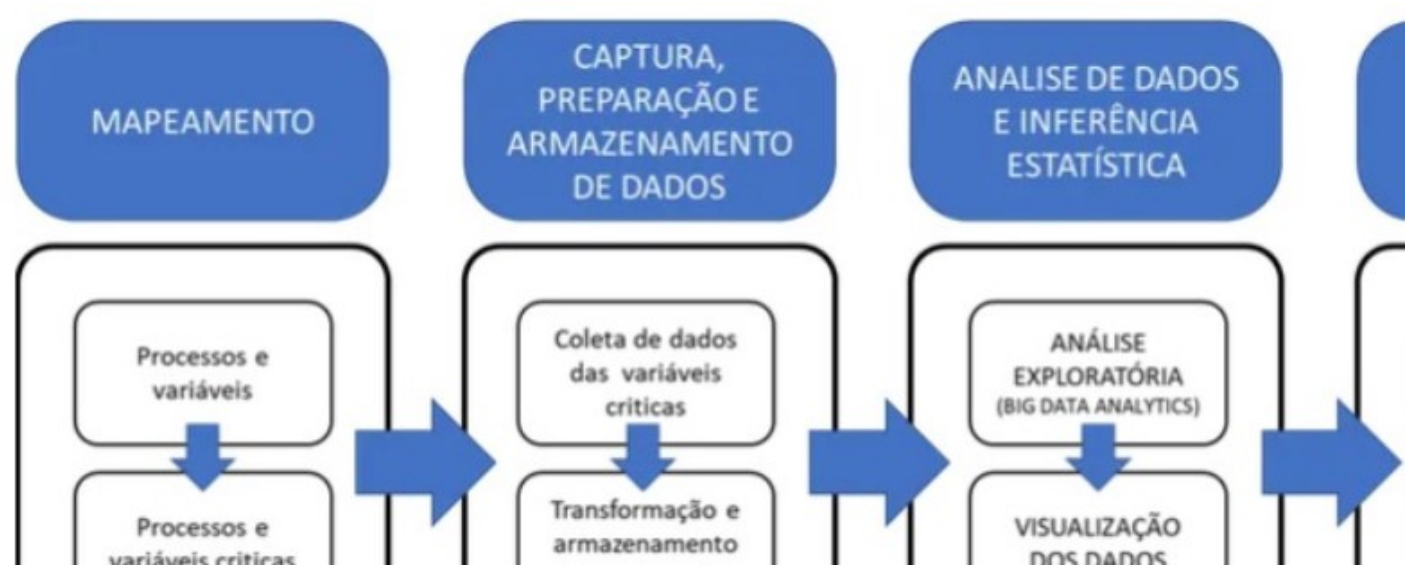

FONTE: AUTORES (2021)

Baseado nos conceitos de "Machine Learning" (Bonaccorso, 2017) e "Inteligência Artificial", o SmartBox cria uma interface simples, inteligente e dinâmica, entre máquina e operador, mostrando em um PC ou dispositivo móvel, conforme ilustrado na Figura 5, informações relevantes da máquina e do processo, dando condições para tomada de decisão rápida e precisa, e reduzir a perda de produtividade.

FIGURA 5. EXEMPLOS DE DASHBOARD PARA SMART BOX
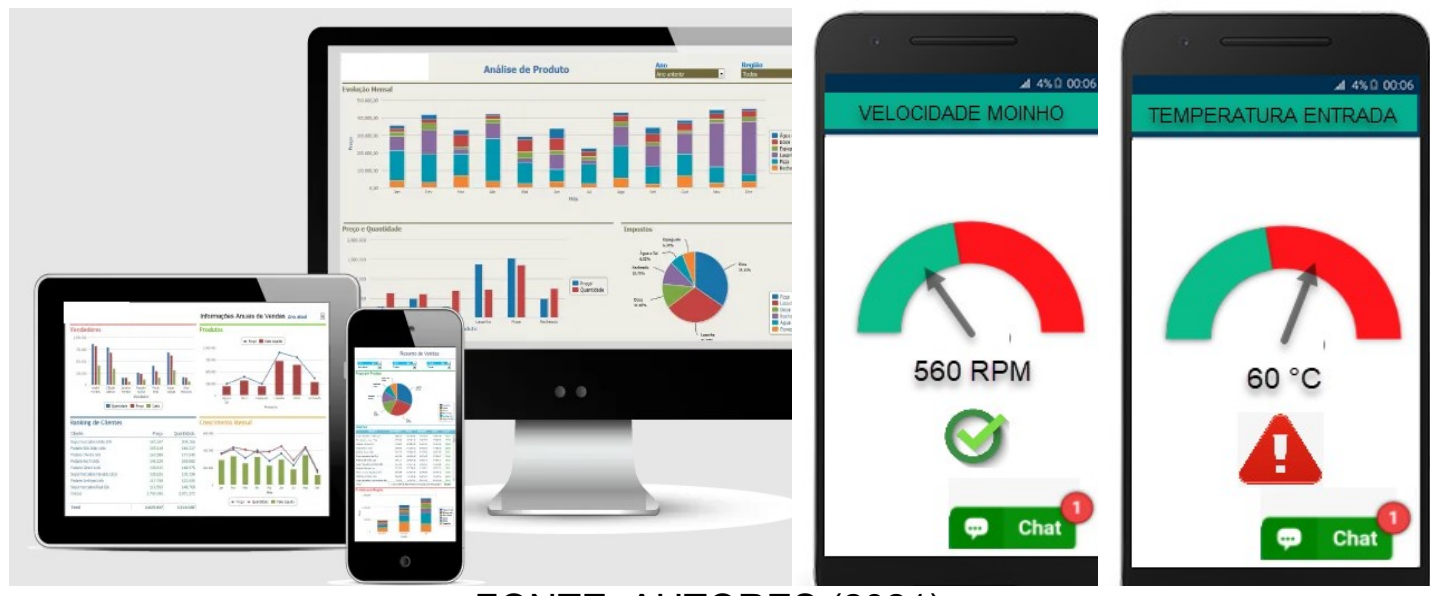

FONTE: AUTORES (2021)

Para o operador, será disponibilizado indicadores simples, que mostram se a máquina está trabalhando dentro do intervalo ideal para o processo.

Como mostrado na seção anterior, o hardware baseado no microprocessador Arduino e a programação em linguagem $\mathrm{C}++$ permite que a lógica de controle 
armazene e processe dados em tempo real, de modo a garantir que a tomada de decisão seja durante o processo e não somente na próxima batelada conforme modelo atual.

\section{METODOLOGIA E PLANEJAMENTO EXPERIMENTAL}

\subsection{ESTUDO DE CASO}

Como base para este estudo de caso foi avaliado uma série de produções (lotes ou bateladas) de tinta eletrostática em pó de um produto específico denominado PWB073 por um período de 14 meses (de janeiro de 2020 até fevereiro de2021), na fábrica PVT INDÚSTRIA DE TINTAS LTDA, localizada no município de Caxias do Sul/RS, Brasil, no equipamento MOINHO DE TINTA A PÓ MODELO LYF60 FABRICANTE YANTAI ANO DE FABRICAÇÃO 2008.

\subsection{PROJETO: LEVANTAMENTO E OBSERVAÇÃO}

O início do trabalho ocorreu em fevereiro de 2020, e foi realizado através de uma série de levantamentos manuais realizados pelos operadores da máquina, das principais variáveis de interesse do processo, da máquina e do ambiente onde a máquina está instalada.

A metodologia de coleta de dados adotada foi registro manual em planilha própria realizada pelos operadores do equipamento no decorrer de cada produção, onde a cada determinada quantidade de caixas (leia-se $25 \mathrm{Kg}$ de material para cada caixa). O operador retira uma amostra para avaliação de tamanho de partícula e anota os dados das configurações do equipamento naquele momento, registra também a hora de início e de fim da batelada. De posse destes dados, foi calculada a Produtividade Média (Kg/h) para cada mês.

\subsubsection{REQUISITOS DO PROJETO}


Deve-se ressaltar as observações abaixo, no que diz respeito aos dados coletados e não coletados, e que são relevantes para este estudo:

a) Temperatura de entrada de ar: não foi avaliada em nenhuma das situações por não ter sido registrada pelos operadores e por não ter sido instalado na máquina um aparelho para medição precisa e dedicada;

b) Temperatura de Saída do Material: foi usado como lastro para aumento de produção, medida em graus Celsius $\left({ }^{\circ} \mathrm{C}\right)$;

c) Parâmetros de Máquina: principais acionamentos (Alimentador, Disco e Classificador), medidos em Hertz (Hz);

d) Produtividade: calculada em Quilograma por hora $(\mathrm{Kg} / \mathrm{h})$, sendo avaliado produtividade real e não instantânea;

e) Valor de Amperagem: Foi convencionado chamar assim o parâmetro para referência da regulagem da velocidade do motor principal (disco) e medido em Amperes (A). Observou-se que este parâmetro é diretamente proporcional a Temperatura de Saída de Material;

f) Velocidade do Ar: não foi considerada, por não ter sido instalado um aparelho ou sensor para medição dedicada na máquina;

g) Temperatura Ambiente: foi considerada pelo registro disponibilizado pelo site https://www.tempo.com/caxias-do-sul-sactual.htm .

\section{RESULTADOS E DISCUSSÃO}

Posteriormente foi realizado o tratamento, filtro e análise dos dados por especialista do processo, que pôde mensurar determinados ajustes (setups) de máquina, verificar e propor novos ajustes.

Em alguns meses houve mais de uma produção (lote), as quais foram compiladas em apenas uma resultante por mês.

Conforme demonstrado na Figura 6, foram destacadas algumas grandezas específicas para a avaliação de melhora do processo de moagem. São elas:

h) Velocidade do Alimentador de matéria prima no equipamento, medido em $(\mathrm{Hz})$; 
i) Velocidade do Disco de Moagem, medido em ( $\mathrm{Hz})$;

j) Velocidade do Classificador, também medido em Hertz $(\mathrm{Hz})$.

FIGURA 6 - GRÁFICO DE ANÁLISE TOTAL DA PRODUTIVIDADE VS. SETUP DE MÁQUINA

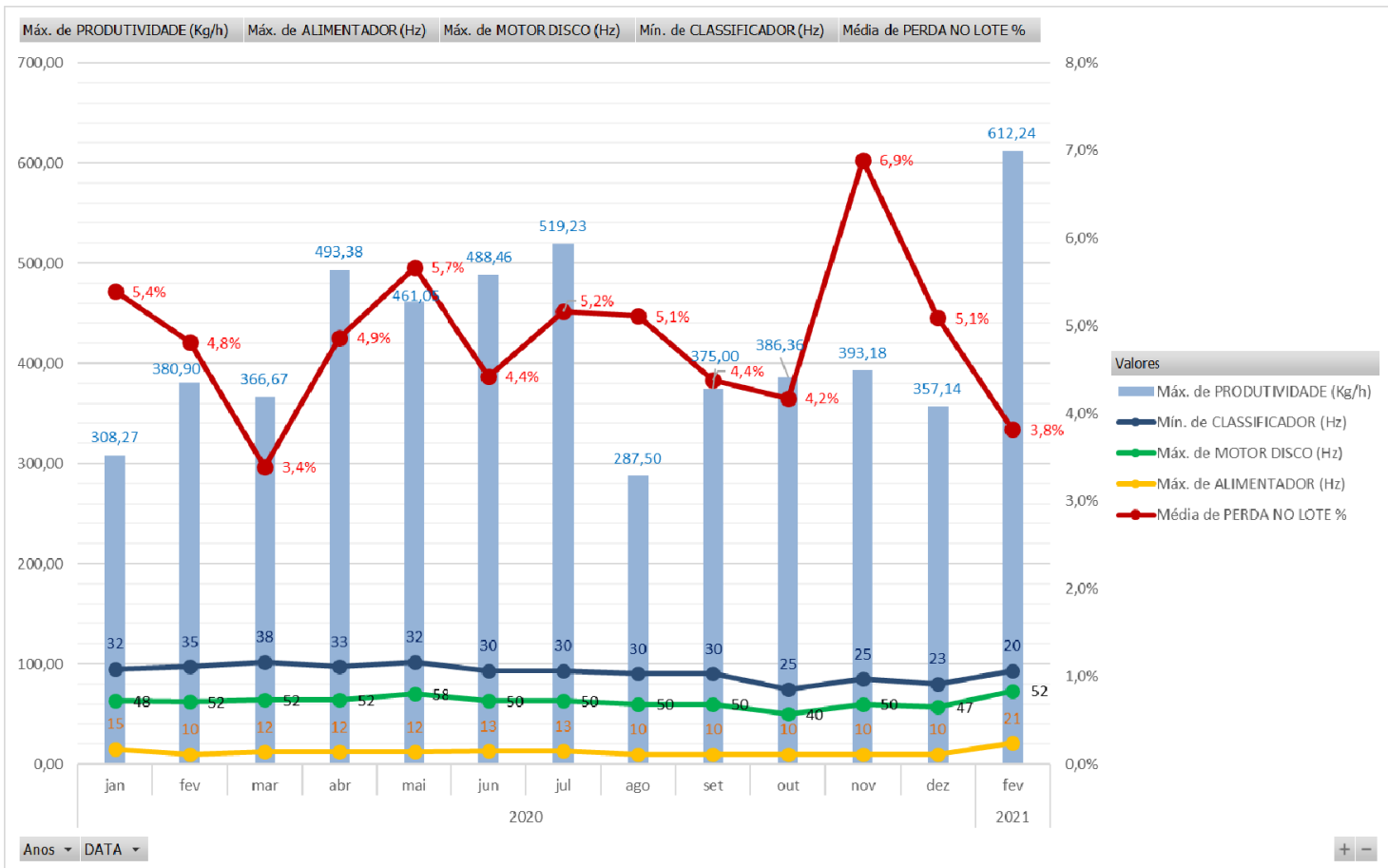

FONTE: AUTORES (2021)

O gráfico demonstra as variações de regulagens de produção (produtividade, classificador, motor disco, alimentador e perda). Pode-se observar que não existe uma linearidade entre as grandezas apresentadas, porem observa-se que quando foi encontrada uma regulagem mais sinérgica entre as variáveis (fev 2021) foi obtido ganho de produtividade e também redução de perda durante o processo.

O segundo lote do mês de fevereiro de 2021 foi o objeto de análise e revisão pelo Gerente de Fábrica, de forma manual, no qual foram feitas alterações de 
configurações para se atingir uma melhor regulagem, a melhor produtividade e a menor perda, conforme demonstrado na Tabela 1.

\section{TABELA 1 - MÊS DE FEVEREIRO DE 2021, COM O SEGUNDO LOTE SENDO OBJETO DE ESTUDO}

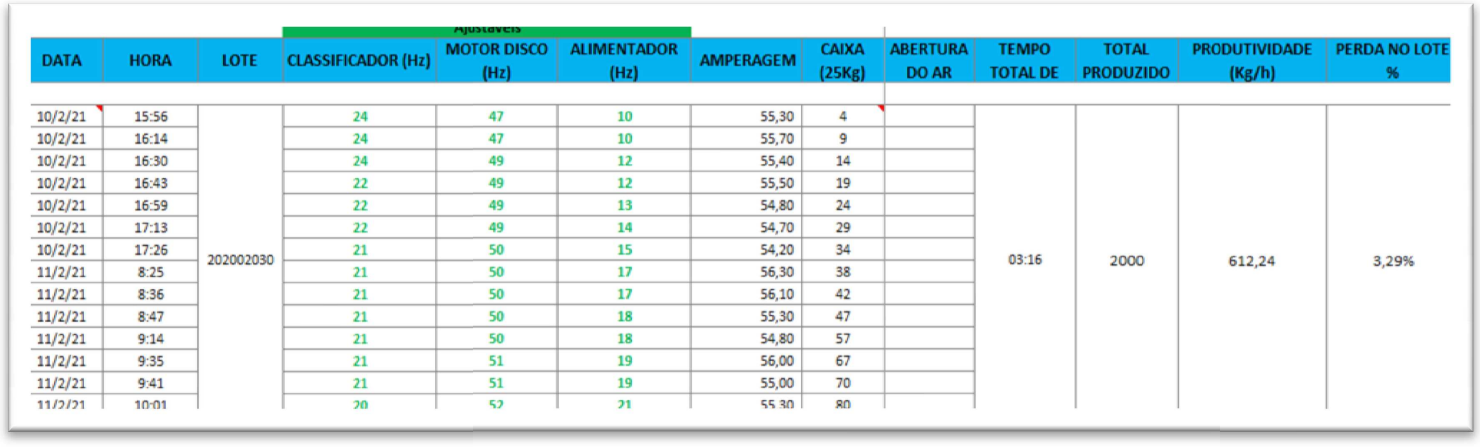

FONTE: AUTORES (2021)

Outro dado muito importante para a avaliação de produtividade do Moinho, é a Perda de Processo. Esta perda é intrínseca ao processo de moagem de tinta eletrostática em pó, ela é proveniente do excesso de moagem do produto, o qual fica muito leve e fino, diminuindo a qualidade do produto final. Devido a isso, este pó fino é separado do pó com tamanho de partícula ideal. Quando há uma má regulagem do sistema de moagem, há também uma tendência a se gerar maior quantidade de material fino ou perda.

A análise do banco de dados levantado comprova que a falta de monitoramento instantâneo e a falta de dados para gerar um melhor setup para o equipamento, geram inconstância de produtividade, que é refletida em perdas. Esta variação, por sua vez, gera uma inconstância no custo do material, refletindo diretamente na margem de contribuição do produto, seja por variação de tempo de máquina e/ou perda de processo. Também acarreta falta de previsibilidade produtiva, fazendo com que o setor de Planejamento e Controle de Produção e Comercial tenham dificuldades em estimar prazos de entrega para os clientes finais. $\mathrm{Na}$ Tabela 2 observamos um exemplo do mês de abril de 2020, no qual foram produzidos dois lotes distintos, onde foram utilizados setups diferentes para cada lote, gerando assim diferentes produtividades e diferentes perdas de processo. 
TABELA 2 - MÊS DE ABRIL DE 2020: DOIS LOTES, DIFERENTES SETUPS, DIFERENTES RESULTADOS.

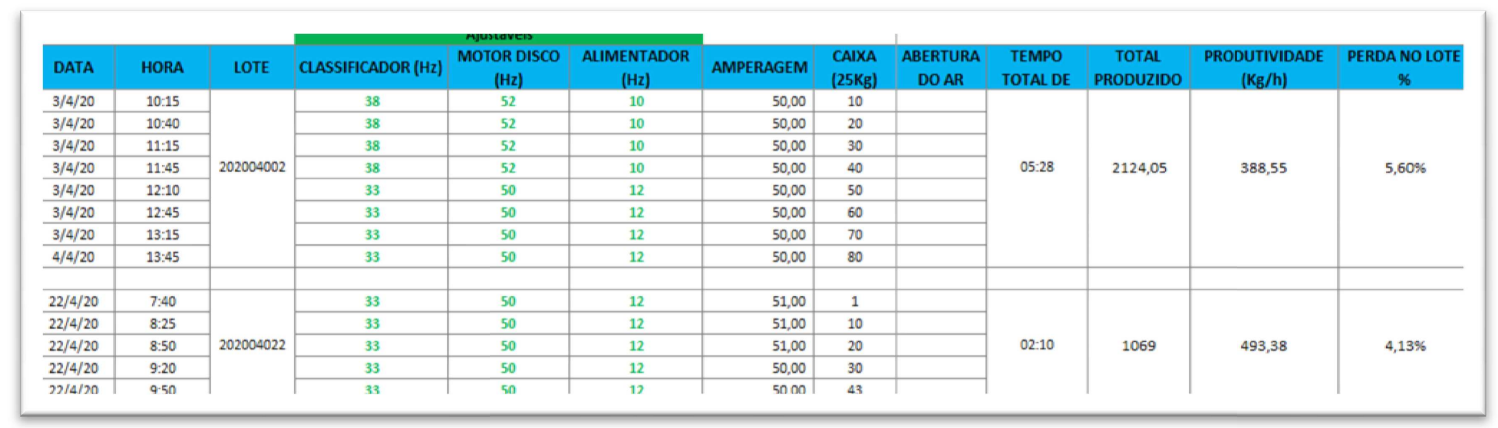

FONTE: AUTORES (2021)

Cruzando-se os dados coletados dos lotes com a rotina do especialista de processo, observou-se que quando há intervenção, seja ela diretamente na regulagem ou apenas pela presença próxima ao operador, a produtividade aumenta!

\section{CONCLUSÕES}

Com base nos resultados apresentados, comparando-se base total de dados históricos (24 lotes), os quais foram produzidos sem monitoramento e registrados pelos operadores, com o lote monitorado e com alterações de ajuste (setup), pode-se observar na Tabela 3 que o ganho de produtividade foi de 59,03\%, bem como constatou-se uma redução na perda na ordem de $32,16 \%$.

TABELA 3 - GANHO DE 59,03\% DE PRODUTIVIDADE E 32,16\% DE GANHO NA PERDA DE MATERIAL.

\begin{tabular}{|l|c|c|c|}
\cline { 2 - 4 } & BASE DE DADOS & LOTE ESTUDADO & GANHO \\
\hline PRODUTIVIDADE MÉDIA (Kg/h) & 384,99 & 612,24 & $59,03 \%$ \\
\hline PERDA MÉDIA (\%) & $4,85 \%$ & $3,29 \%$ & $32,16 \%$ \\
\hline
\end{tabular}

FONTE: AUTORES (2021) 
Neste lote monitorado os parâmetros foram alterados durante o processo, objetivando-se o melhor ajuste (setup) para o equipamento, mantendo-se sempre o tamanho de partícula, ou seja, respeitando-se as especificações do produto. O lote começou com menor produção e foi aumentado gradativamente. Ao analisar a produtividade do último ajuste (setup), observamos que o equipamento produziu 10 caixas (ou $250 \mathrm{Kg}$ ) em 20 minutos, o que resultou em uma produção total de $750 \mathrm{Kg} / \mathrm{h}$, ou seja, ganho de $94,81 \%$ de produtividade. Esta configuração só foi atingida devido ao histórico gerado pelos dados coletados no equipamento, como temperaturas, rotações dos acionamentos e tamanho de partícula, o que gerou a confiança para as alterações nas regulagens.

Podemos, portanto, comparar a produção por mês de um equipamento sem o Smartbox e com o Smartbox, levando-se em consideração 8horas ininterruptas diárias de trabalho, durante 20 (vinte) dias úteis - conforme Tabela 4 abaixo:

\section{TABELA 4 - COMPARATIVO DE PRODUÇÃO “COM” E "SEM" SMART BOX}

\begin{tabular}{|c|c|c|c|} 
& $\begin{array}{c}\text { MÉDIA } \\
\text { PRODUÇÃO / h }\end{array}$ & $\begin{array}{c}\text { PRODUÇÄO } \\
\text { MÊS (Kg) }\end{array}$ & $\begin{array}{c}\text { GANHO } \\
(\%)\end{array}$ \\
\hline sem SmartBox & 385 & 61600 & \\
\hline com SmartBox & 750 & 120000 & $94,81 \%$ \\
\hline
\end{tabular}

FONTE: AUTORES (2021)

Além da eficiência comprovada através dos resultados apresentados, deve-se também enfatizar o fator de custo do SmartBox, que pode ser considerado de baixo custo, quando comparado com o ganho que pode prover. O custo total do Hardware é estimado em $\mathrm{R} \$ 700,00$.

Baseados nestes dados pode-se concluir que quando conhecemos melhor as variáveis de processo e as monitoramos em tempo real, tem-se as condições para se atingir o maior nível de produtividade, com menores perdas e consequente redução dos custos de processo.

Por comparação entre os resultados, pode-se ainda concluir que o uso da Smart Box poderá agregar valor na tomada de decisão operacional para o melhor ajuste (setup) e ser utilizada como interface auxiliar na padronização operacional. 
Os resultados demonstrados comprovaram a melhora do processo com menores perdas, ganho de produção e padronização operacional.

\section{REFERÊNCIAS BIBLIOGRÁFICAS}

AVELAR, C. F. Mineração de dados, Instituto Vianna Júnior, Juiz de Fora MG, 2017

BONACCORSO, G. Machine Learning Algorithms, Editora: Packt Publishing 360 páginas 2017

ERIDANI, D. Pengenalan dan Analisis Ucapan pada Sistem Kontrol Perangkat Listrik Meinggunakan Arduino Uno. Diponegoro University, Indonesia 2018

MISEV, T.A. POWDER COATINGS Chemistry and Tecnhology - DSM Resins - by John Wiley \& Sons Ltd., 1991

ROSA, J. L. G. Fundamentos da Inteligência Artificial, Rio de Janeiro, 2011

THOMAZINI, D. P. B. A. Sensores Industriais: FUNDAMENTOS E APLICAÇÕES. ERICA, 9a Edição, São Paulo, 2020

PAIVA, J. M. F., MAYER, S., CÂNDIDO, G. M., REZENDE, M. C. Avaliação da temperatura de transição vítrea de compósitos poliméricos reparados de uso aeronáutico. 2006

\section{AGRADECIMENTOS}

Agradecemos aos nossos professores do curso, orientadores, especialmente ao Professor Alessandro Marques, e aos gerentes e diretores da fábrica PVT INDÚSTRIA DE TINTAS LTDA por permitirem este estudo e acesso em suas instalações, laboratórios e equipamentos. 